TAP CHÍ KHOA HOC ĐÄI HỌC TÂN TRÀO
ISSN: $2354-1431$

\title{
ĐặC ĐIỂM NGÔN NGŨ̉ MẠNG CỦA SINH VIÊN NHÌN TỪ BÌNH DIỆN CÂUU TRÚC (QUA MỘT SỖ DIỄN ĐÀN)
}

\author{
TS. Nguyến Thị Hồng Chuyên ${ }^{1 *}$; SV. Nguyến Thị Quỳnh Hoa ${ }^{l}$; SV. Bùi Thị Mai Hurong ${ }^{1}$ \\ ${ }^{1}$ Truoòng Đại học Tân Trào \\ *Email: hongchuyennnvn.tn@gmail.com
}

\section{Thông tin bài viết}

Ngày nhận bài:

$29 / 6 / 2020$

Ngày duyệt đăng:

20/9/2020

Từ khóa:

ngôn ngũ mạng, sinh viên,

bình diện cấu trúc, diễn

đàn.

\section{Tóm tắt}

Bài báo dựa trên các lí luận liên quan đến phương ngữ xã hội và ngôn ngữ mạng; sinh viên và bối cảnh văn hoá để tìm hiểu đặc điểm ngôn ngữ mạng của sinh viên ( $\mathrm{SV}$ ) từ bình diện cấu trúc ở các khía cạnh như: tiếng lóng, chêm xen ngôn ngữ thứ hai trong tương tác, kết cấu mới. Từ kết quả nghiên cứu, chúng tôi nhận định: Ngôn ngữ mạng của $S V$ trên bình diện cấu trúc thể hiện sự năng động, sáng tạo, dám nghĩ, dám làm của tuổi trẻ và sự vận động của thời đại được thể hiện qua ngôn ngữ của sinh viên.

\section{1. Đặt vấn đề}

Ngôn ngữ là phương tiện giao tiếp quan trọng nhất của con người. Cùng với sự phát triển, biến đổi của văn hoá xã hội và thời đại, ngôn ngữ cũng không ngừng biến đổi theo để thực hiện chức năng của mình.

Trong bối cảnh hội nhập diễn ra mạnh mẽ và sự bùng nổ của khoa học công nghệ, tiếng Việt trong sử dụng đang có sự biến đổi sâu sắc trên nhiều phương diện từ hình thức từ ngữ âm, ngữ pháp, ngữ nghĩa, ngữ dụng. Sự biến đổi đó không đồng nhất giữa các vùng miền, các đối tượng. Vì vậy, tạo nên rất nhiều các phương ngữ xã hội (PNXH) khác nhau. Hiện nay, một trong những kiểu $\mathrm{PNXH}$ được nhắc đến trong tiếng Việt là phương ngữ theo tuổi tác mà ngôn ngữ của giới trẻ được chú ý nhiều nhất. Trong ngôn ngữ của giới trẻ, chúng tôi quan tâm đến ngôn ngữ của sinh viên - đây là nhóm có số lượng đông, nhanh nhạy với cái mới, thích khám phá, ưa tìm tòi, sáng tạo nên luôn tạo ra những trào lưu xã hội mới.

Mạng xã hội là một trong những kênh truyền thông phục vụ cho đời sống của xã hội. Ngày nay, dưới sự phát triển mạnh mẽ của thời kì Cách mạng công nghệ 4.0 và điều kiện vật chất của người dân được cải thiện rõ rệt, việc sử dụng các thiết bị công nghệ tiến tiến và những kênh truyền thông đa phương tiện ngày càng phổ biến. Đây là nhân tố tạo nên các cộng đồng ảo với tốc độ truyền tin cực lớn và điều này kéo theo sự biến đổi sâu sắc tiếng Việt.

Trong phạm vi bài viết, chúng tôi tìm hiểu đặc điểm ngôn ngữ mạng của sinh viên từ bình diện cấu trúc gồm: tiếng lóng, chêm xen ngôn ngữ thứ hai trong tương tác, kết cấu mới.

Nguồn tư liệu được chúng tôi xác định là một số diễn đàn của sinh viên như: sinhvienfanpage; KenhSV.vn; hoisinhvien.com.vn; vnu.ulis; Đoànthanh-niên-Hội-sinh-viên-trường-Đại-Học-Tân-Trào; honghotsinhvien.

\section{Nôi dung nghiên cứu}

\subsection{Một số vấn đề lí luận liên quan}

\subsubsection{Phương ngữ xã hội và ngôn ngữ mạng}

Phương ngũ̃ xã hội (PNXH) là một trong những nội dung quan trọng của Ngôn ngữ học xã hội. Theo 
Fasol (1990), PNXH là những cách nói năng của các nhóm dân cư cùng hoạt động trong một lĩnh vực kinh tế, thuộc cùng một giai tầng xã hội, cùng thế hệ, tuổi tác, đẳng cấp tôn giáo [1]. Tác giả Nguyễn Văn Khang quan niệm PNXH là sản phẩm ngôn ngữ của nhóm xã hội. Các nhân tố xã hội như: tuổi tác, giới tính, nghề nghiệp, trình độ văn hoá, thành phần xuất thân,...có tác động trực tiếp và tạo nên các đặc điểm ngôn ngữ trong sử dụng. Hay, PNXH là các biến thể ngôn ngữ theo nhóm xã hội trong sử dụng ngôn ngữ. [2, tr. 214]

Như vậy, có thể hiểu PNXH là các biến thể ngôn ngữ của nhóm xã hội dưới tác động của các nhân tố xã hội như: tuổi tác, giới tính, nghề nghiệp, trình độ văn hoá,... Trong bài viết, chúng tôi quan niệm, ngôn ngữ mạng của sinh viên là một biến thể ngôn ngữ của nhóm sinh viên trong tiếng Việt hiện nay.

"Ngôn ngũu mạng là ngôn ngũ đặc thù của phuơng ngũ cá nhân của các cu dân mạng và của cộng đồng mạng nói chung, tùng cộng đồng mạng cu thể nói riêng" [3]. Điều đáng lưu ý, mặc dù là phương ngữ cá nhân của các cá nhân với phong cách riêng nhưng ngôn ngữ mạng lại được "xã hội hoá" thông qua mạng xã hội (Fb, Sky, Zalo, Twitter,...) và thoại trường giao tiếp của ngôn ngữ mạng đặc biệt thế giới "ảo".

Trong bài viết, chúng tôi quan niệm, ngôn ngữ mạng của sinh viên là một biến thể ngôn ngữ của nhóm sinh viên trong tiếng Việt hiện nay.

\subsubsection{Sinh viên và bối cảnh văn hoá của sinh viên}

Theo quy định của Quốc hội nước Cộng hòa xã hội chủ nghĩa Việt Nam tại Luật Giáo dục đại học (2012), quy định "người đang học tập và nghiên cúu khoa hoc tại co sở giáo dục đại họ, gồm sinh viên của chuơng trình đào tạo cao đẳng, chuoong trình đào tạo đại hoc" [4]. Có thể hiểu, sinh viên là người đang học tập và nghiên cứu khoa học tại các cơ sở giáo dục đại học đào tạo chương trình cao đẳng, chương trình đại học.

Từ quy định trên, trong bài viết chúng tôi xác định: sinh viên là những người đang theo học tại các trường đại học, cao đẳng; có độ tuổi khoảng từ 18 đến 25 (độ tuổi bắt đầu bước vào chương trình cao đẳng hoặc đại học cho đến khi tốt nghiệp). Do phạm vi tương tác và nhận thức ở giai đoạn này có sự biến đổi mạnh nên SV là nhóm xã hội luôn có sự biến động theo hướng: tiếp thu các tinh hoa, kiến tạo các tri thức mới, mang trong mình sứ mệnh kế thừa và phát triển dân tộc. Tuy nhiên, do sự biến động này $\mathrm{SV}$ chưa có được các bản sắc, tính bền vững, ổn định. Đặc biệt, nhóm xã hội là sinh viên được tập hợp do tiêu chí độ tuổi chi phối nên làm cho nội nhóm có sự phân hoá về: xuất thân, giới tính, trình độ học vấn, tôn giáo, phong tục tập quán,... Như vậy, sinh viên là nhóm xã hội có tính phức hợp cao và chịu tác động của rất nhiều các nhân tố xã hội.

Trong dòng chảy chung của nền văn hoá dân tộc, văn hoá thanh niên (trong đó có sinh viên) là một trong những bộ phận nổi trội bởi trong đời sống xã hội, văn hoá thanh niên luôn dẫn đầu các trào lưu, hiện tượng mới mẻ, tạo ra các giá trị lệch chuẩn truyền thống và thường gây xung đột, tranh cãi. Sinh viên nói riêng và thanh niên nói chung trong thời kì hội nhập sâu và rộng như hiện nay lối tư duy, hành động, cách thể hiện khác thế hệ trước rất nhiều; đôi khi họ cố tình tạo ra, làm trái với quy luật thông thường để thể hiện mình.

Như vậy, dựa trên các cơ sở lí luận vừa nêu, chúng tôi tiến hành khảo sát, thống kê, phân loại và miêu tả các đặc điểm của ngôn ngữ mạng của sinh viên trên một số diễn đàn

\subsection{Kết quả khảo sát thông kê}

Từ phạm vi tư liệu được xác định là một số diễn đàn của sinh viên như: sinhvienfanpage; KenhSV.vn; hoisinhvien.com.vn; vnu.ulis; Đoàn-thanh-niên-Hộisinh-viên-trường-Đại-Học-Tân-Trào; honghotsinhvien, chúng tôi tiến hành khảo sát ngữ liệu.

Tiêu chí xác định ngôn ngữ mạng của sinh viên là ngôn ngữ do nhóm xã hội sinh viên sử dụng trong các diễn đàn lựa chọn trên và được phân loại theo 3 nhóm: 1/ là tiếng lóng (từ ngữ lóng); $2 /$ chêm xen ngôn ngữ thứ hai trong tương tác (ngoại ngữ, tiếng mẹ đẻ - đối với SV người dân tộc thiểu số); 3/ các kết cấu mới lạ.

Bảng 1. Bảng khảo sát và phân loại các hình thức của ngôn ngữ mạng của sinh viên

\begin{tabular}{|c|c|c|c|c|}
\hline $\begin{array}{l}\text { Tiêu chí } \\
\text { xác định }\end{array}$ & Số lượng & Tỉ lệ (\%) & Ví dụ & Ngũ liệu \\
\hline $\begin{array}{l}\text { Tiếng lóng } \\
\text { (từ ngữ lóng) }\end{array}$ & 97 & $48.5 \%$ & $\begin{array}{l}\text { giờ cao su, a cay, vãi, khủng, } \\
\text { chuối, rụng tim, thánh, sư phụ, } \\
\text { ngáo, lầy, vãi chưởng, ngống, } \\
\text { cúp giờ, nhảy tiết, Pà cô, tám, } \\
\text { phê lòi,... }\end{array}$ & $\begin{array}{l}\text { - Su phụ chỉ em bài này } \\
\text { với! } \\
\text { - Chiều nay nhảy hai tiết } \\
\text { đầu nhá. } \\
\text { - Bài hôm nay chuối quá. }\end{array}$ \\
\hline
\end{tabular}




\begin{tabular}{|c|c|c|c|c|}
\hline $\begin{array}{l}\text { Tiêu chí } \\
\text { xác định }\end{array}$ & Số lượng & Tỉ lệ (\%) & Ví dụ & Ngữ liệu \\
\hline $\begin{array}{c}\text { Chêm xen } \\
\text { ngôn ngữ thứ } 2\end{array}$ & 72 & $36 \%$ & $\begin{array}{l}\text { sorry, no, clip, miss, team, } \\
\text { style, book, inbox, book, chat, } \\
\text { cute, thank you, hotgirl, } \\
\text { hotboy, drama, view, idol,... }\end{array}$ & $\begin{array}{l}\text { - Vào cày view cho idol đi } \\
\text { các bạn ơi. } \\
\text { - Iu và nhớ team nè. } \\
\text { - Sorry chị! }\end{array}$ \\
\hline Kết cấu mới & 31 & $15.5 \%$ & $\begin{array}{l}\text { Tay nhanh hơn não, não quên ở } \\
\text { nhà, trượt vỏ chuối, diện kiến } \\
\text { trưởng khoa, ảo tưởng sức } \\
\text { mạnh, ngất trên cành quất,... }\end{array}$ & $\begin{array}{l}\text { - Đọc kĩ thông báo! Tay } \\
\text { nhanh hơn não à? } \\
\text { - Không học mà đòi A! Ao } \\
\text { tướng súc mạh quá. } \\
\text { - Qua thằng Hoàng được } \\
\text { diện kiến trương khoa } \\
\text { đấy. }\end{array}$ \\
\hline Tổng số & 200 & $100 \%$ & & \\
\hline
\end{tabular}

Dựa theo bảng 1 , chúng tôi đưa ra một số nhận định:

- Tổng số ngữ liệu thu được là 200, trong đó: 1/ tiếng lóng (từ ngữ lóng) có 97/200 đơn vị, chiếm $48.5 \%$; 2/ chêm xen ngôn ngữ thứ hai có 72/200 đơn vị, chiếm 36\%; 3/ Kết cấu mới 31/200 đơn vị, chiếm $15.5 \%$.

- Số lượng đơn vị là tiếng lóng chiếm số lượng lớn nhất trong các nhóm còn lại đã phản ánh mục đích, thái độ, đặc trưng của nhóm SV. Hay, tiếng lóng là đặc trưng của ngôn ngữ nhóm $\mathrm{SV}$; đồng thời, thể hiện tính năng sản của ngôn ngữ. Số lượng các từ chêm xen chiếm số lượng thứ 2 , điều này phản ánh sự tương tác của xã hội diễn ra đa chiều. Trong giao tiếp, $\mathrm{SV}$ bên cạnh việc sử dụng tiếng Việt còn sử dụng tiếng dân tộc thiểu số của mình và ngoại ngữ (Tiếng Anh). Nhóm kết cấu mới lạ chiếm số lượng ít nhất bởi khả năng kết hợp các đơn vị ngôn ngữ tạo thành kết cấu hạn chế.

\section{3. Đặc điểm ngôn ngũ mạng của sinh viên xét tù bình diện cấu trúc}

\subsubsection{Tiếng lóng}

Tiếng lóng là một trong những đặc trưng của ngôn ngữ nhóm SV. Tiếng lóng là sản phẩm đặc thù của mỗi nhóm xã hội và được tạo nên từ "vật liệu" là từ ngữ lóng. Đối với nhóm xã hội là $\mathrm{SV}$, tiếng lóng được xác định là các từ ngữ được các cá nhân trong nhóm sử dụng trong nội nhóm, thể hiện phong cách và ngôn ngữ của nhóm.

Từ kết quả khảo sát, chúng tôi xác định 97 từ ngữ lóng, tiêu biểu như: giờ cao su, a cay, vãi, khủng, chuối, rụng tim, thánh, ngáo, lầy, vãi chưởng, ngỗng, cúp giò̀, Pà cô, tám, phê lòi, ccmnr, FA, ib, ... Trong số các từ ngữ thu được, có những từ ngữ có tần số xuất hiện lớn, trở thành tín hiệu nhận diện cho biến thể ngôn ngữ này như: lầy (10 lần), vãi chuoơng (8 lần), ngáo (8 lần), chuối (7 lần), tám (6 lần),...

a) Về ngữ âm - cấu tạo

Tiếng lóng của SV được hình thành từ nhiều con đường khác nhau. Tuy nhiên, trong phạm vi bài viết, chúng tôi trình bày 3 con đường chính:

Thư nhất, biến đổi những đơn vị có sẵn trong tiếng Việt. Đây là con đường tạo từ chủ yếu mà $\mathrm{SV}$ dùng. Các hình thức biến đổi gồm: biến đổi hình thức ngữ âm; biến đổi ngữ nghĩa.

Biến đổi hình thức ngữ âm được chia thành: 1/ Biến đổi một phần âm tiết nhu: ngâm cứu (nghiên cứu), khoai (khó), túm lại (tóm lại), buôn (ngồi lê đôi mách), Gia Cát Dự (dự đoán),...; 2/ Đồng âm khác nghĩa: đảo ngói (đảo ngôi tóc), bồng bềnh (béo), Pà cô (cô giáo), buôn (ngồi lê đôi mách), ...; 3./ Nói lái: chống lầy, hại điện, bật mí, xịn xò, ... Ví dụ:

\section{(1) - Đợt tới đi tình nguyện chứ?}

- Ngâm cứu đã.

(2) Đề thi năm nay khoai quá !

Biến đổi nghĩa để tạo nên tiếng lóng của SV. Các các biến đổi nghĩa như: 1 / cấp thêm nghĩa mới cho từ: gấu (nguyên nghĩa: thú ăn thịt cỡ lớn, đuôi cộc, đi bằng bàn chân, thường trèo cây ăn mật ong/ hung dữ và hỗn láo - nghĩa chuyển: người yêu), hột me (nguyên nghĩa: hạt của quả me - nghĩa chuyển: ý thức kém), não phẳng (nguyên nghĩa: não (khối tập trung các thần kinh trung ương nằm trong sọ không thành nếp), phẳng (bề mặt bằng, đều, không lồi lõm) - nghĩa chuyển: ý thức kém), $K$ (nghìn),..; 2/ thu 
hẹp nghĩa gốc: bá đạo (nguyên nghĩa: chính sách của kẻ dựa vào vũ lực, hình phạt, quyền thế mà thống trị ở thời phong kiến Trung Quốc cổ đại - nghĩa chuyển: tính chất hay, hấp dẫn, độc đáo với mức độ cao), khủng (nhiều hơn mức bình thường - học bổng khủng), tiến bối (người đi trước), ... Ví dụ:

(3) Cố lên! Học bổng khủng thế cơ mà.

Thứ hai, tạo từ mới. Đây là cách giới trẻ sử dụng những vật liệu và phương thức tạo từ vốn có của tiếng Việt để tạo nên từ ngữ mới. Ví dụ: bồ kết (thích), vã (muốn), cúp giờ (trốn học), ngáo (không hiểu), quẩy (chơi), bựa/ nhây (không dứt khoát), thả thính/ rắc thính (tán gán/ trai), đu đưa (đi chơi), ... Ví dụ:

(4) Thi xong rồi! Quẩy thôi chiến hữu ơi!

Thứ ba, vay mượn từ ngoại lai. Đây là con đường vay mượn các đơn vị từ vựng lẫn cách thức cấu tạo từ ngoại lai. Những đơn vị từ vựng này hoàn toàn không có trong tiếng Việt toàn dân. Chẳng hạn: G9, F5, @, soái ca, soái tỷ, tiền bối,... Ví dụ:

(5) Ngày mai thi tốt! G9!

(6) Soái ca khoa mình có người yêu rồi! Tiếc quá!

b) Về ngữ nghĩa

Tiếng lóng của SV là một hình thức biểu hiện đặc sắc và điển hình về bình diện ngữ nghĩa, thể hiện: tính đa nghĩa, tính biểu thái.

- Tính đa nghĩa là một hiện tượng phổ quát của nhiều ngôn ngữ và tiếng Việt cũng mang đặc tính này. Xem xét tính đa nghĩa của các từ ngữ khảo sát, chúng tôi quan niệm: các từ ngữ lóng có nhiều hơn một nghĩa và các nghĩa có liên quan với nhau. Kết quả cụ thể:

+ Đa nghĩa do phái sinh nghĩa lóng từ nghĩa gốc: nai, gấu, gạch đá, ngon, lúa, lầy, nhọ, đặc sản,...

Đây là nhóm từ ngữ lóng có số lượng lớn. Lí do, nhóm này được hình thành từ đơn vị có sẵn và được chuyển đặc điểm nào đó trong nghĩa để tạo nên từ ngữ lóng. Nói cách khác, đây là nhóm được hình thành do sự chuyển nghĩa và chủ yếu là bằng hai phương thức ẩn dụ và hoán dụ. Chẳng hạn: săn/săn học bổng, phao, ruột mèo, cửa, tủ,... Ví dụ:

(7) Năm tới săn lấy vài ba cái học bổng là ok.

(8) Làm cho tao bộ ruột mèo nhá.

+ Đa nghĩa lóng tức là tự thân nghĩa lóng gồm nhiều nét nghĩa khác nhau ngoài nghĩa gốc, chẳng hạn: trâu, xoắn, ảo, nóng, đo, hàng, chảnh, pin trâu, A Phủ,... Ví dụ:
Từ lóng "trâu" có các nghĩa như sau:

(9) Ngày xưa trẻ trâu vãi. (trẻ trâu = trẻ chăn trâu)

(10) Năm thứ 3 rồi mà còn hành động vậy. Trẻ trâu. (trẻ trâu = hành động nông nổi, bồng bột.)

(11) Oài! trâu! (trâu = khoẻ)

- Tính biểu thái của từ ngữ lóng của sinh viên là ý nghĩa biểu hiện thái độ vui tươi, hài hước, dí dỏm bởi đặc điểm tuổi tác, thái độ của SV quy định. Ví dụ: le vồ, ô sò kê, ai lớp viu, xì tin, gấu, ngáo, tỉnh tò, tụ suớng, hạt dẻ, đá,... Tính biểu thái được tạo ra bởi nhiều lí do: do hiện tượng chêm xen tiếng Anh hoặc do sự liên tưởng giữa nghĩa gốc và nghĩa lóng. Ví dụ:

(12) - Tối nay đi chơi nha?

- Ô sò̀ kê nhá!

(13) - Hộ tao tí?

- Hộ gì?

- Vào gánh Team hộ tao. Tao đi vệ sinh.

\subsubsection{Chêm xen ngôn ngũu thứ hai trong tuoong tác}

Hiện tượng chêm xen ngôn ngữ thứ hai là hiện tượng sử dụng các tư ngữ ngoài tiếng Việt. Hiện tượng này xuất hiện phổ biến trong xu thế hiện nay việc học tập tiếng Anh là bắt buộc đối với sinh viên. Đồng thời, với các SV là người dân tộc thiểu số hoặc sống trong vùng DTTS, hoặc biết tiếng DTTS có thể sử dụng chêm xen tiếng dân tộc mình trong giao tiếp. Đặc điểm chêm xen ngôn ngữ thứ hai trong tương tác ngày càng mở rộng phạm vi do hiện nay xu hướng người đa ngữ, cộng đồng đa ngữ đang ngày càng mở rộng phạm vi. Trong bài viết, chúng tôi trình bày về hiện tượng chêm xen tiếng Anh trong tương tác của SV và đồng thời ghi nhận 72 lượt từ ngữ là tiếng Anh.

a) Về đặc điểm ngữ âm

Hình thức tiếng Anh được SV chêm xen trong giao tiếp thể hiện ở hai dạng: 1/ viết đúng theo hình thức chuẩn của tiếng Anh; $2 /$ viết theo các phỏng âm của người Việt, cụ thể:

+ Hình thức viết theo đúng chuẩn tiếng Anh như: Ok, thanks, style, video call, team, book, nail, ship, sale, hotboy, hotgirl, inbox, hot, simple love, baby, like, share, makeup, game, idol, event, page, ... Ví dụ:

(14) Style chống liệt môn Sinh.

(15) Share cỏ bốn lá để may mắn trong kì thi sắp tới.

(16) thui thì sn này chắc video call thì kp quà cáp gì nữa uyên nhỉ. 
+ Hình thức viết tiếng Anh theo cách phát âm của người Việt, chẳng hạn: thanh kiu (thanh you), ồ dế (ok), xì tin/ sò tai (style), hót go (hotgirl), hót boi (hotboy), nét (net), đò̀ ra ma (drama), nâu nâu (no no), búc (book), chệch in (check in), gu gồ (google), phây (face),... Ví dụ:

(17) Thanh kiu em yêu! (Thanh kiu = Thank you)

(18) Dạo này lắm đờ $r a m a$ thế. (đờ ra ma drama)

b) Về đặc điểm từ loại

Các từ ngữ lóng mượn từ tiếng Anh chủ yếu ở các từ loại như: danh từ/ cụm danh từ, động từ/ cụm động từ, tính từ/ cụm tính từ. Chẳng hạn: link, email, camera, order, ship, hot, cute, comment, I love you, action,... Ví dụ:

(19) Các bạn sinh viên sẽ action thế nào khi rơi vào hoàn cảnh này?

(20) Cho tớ xin cái link.

(21) I love you. Chiu chiu.

c) Về đặc điểm ngữ nghĩa

Phần lớn các từ ngữ tiếng Anh khi được chêm xen thường giữ nguyên nghĩa gốc. Chẳng hạn: email, link, hotboy, hotgirl, like, share, team, laptop, book, happy, style, shock, hack, .... Ví dụ:

(22 Lập team bắn cá nha.

(23) Share mạnh lên để lớp mình đạt giải. haha

(24) Các fan đâu rồi?

Tuy nhiên, có những từ ngữ tiếng Anh khi được sử dụng có những sự thay đổi so với nghĩa gốc. Sự biến đổi xuất hiện là do người dùng chỉ chọn một nét nghĩa gốc để biểu thị. Chẳng hạn:

Từ style trong tiếng Anh là danh từ với các nét nghĩa như: 1/ loại, thứ phong cách (viết, nói, làm); 2/ nét đặc trưng phong cách (của con người, trường học, thời đại); 3/ danh hiệu, tước hiệu; 4/ phẩm cách, điều đặc biệt; $5 /$ mốt, thời trang; $6 /$ bút (để vạch, khắc) [5, tr.959]. Trong tiếng lóng của $\mathrm{SV}$, style có các nét nghĩa như:

(25) Style quá ngầu.

(26) Cảm nhận hoang mang style.

(27) Xì tin quá gái.

\subsubsection{Kết cấu mới}

Kết cấu mới trong ngôn ngữ mạng của $\mathrm{SV}$ là các kết cấu có cấu tạo là các cụm từ. Đây cũng là một trong hình thức tạo nên ngôn ngữ riêng khác của $\mathrm{SV}$ trên các diễn đàn. Điểm đặc biệt của các kết cấu này đó chính là sự phá vỡ các quy tắc cấu tạo lẫn đặc điểm nghĩa biểu hiện so với các quy tắc truyền thống. Tuy vậy, tính phá vỡ quy tắc lại được chế định và mang lại tính ổn định cho các kết cấu mới là bởi kết cấu được tổ chức tương đối ổn định và theo các phương thức riêng. Theo kết quả khảo sát có 31 kết cấu mới lạ như: tay nhanh hơn não, nhỏ mà có võ, não quên ở nhà, truợt vỏ chuối, diện kiến truởng khoa, ảo tuơong sức mạh, ngất trên cành quất, tuyệt cú mèo, bao phê bao ngầu, thích là nhích, xấu nhu gấu, ngon lành cành đào, rảnh rỗi sinh nông nổi, tủ đè mất xác, bó tay chấm com, nhìn đề câm nín, chân dài não ngắn, ...

a) Về đặc điểm ngữ âm

Các kết cấu mới lạ được cấu tạo đặc biệt và tạo nên các đặc điểm ngữ âm riêng khác. Các cách thức cấu tạo của kết cấu mới lạ về mặt ngữ âm gồm các phương thức như sau: phương thức hiệp vần, phương thức cải biên, phương thức vay mượn, phương thức sáng tạo mới.

- Phương thức hiệp vần là phương thức sử dụng sự bắt vần trong ngôn ngữ để tổ chức lời nói ở cấp độ trên từ mà không lặp lại cấu trúc các tiếng nhằm tạo nên tính liên kết, hài hoà cho lời nói. Chẳng hạn: cao thủ đầu có mủ, ngất trên cành quất, thích là nhích, ngon lành cành đào, chán nhu con gián, hiện đại hại điện, đơn giản nhu đan rổ, chuẩn không cần chinh,... Phương thức này tạo nên tính hài hoà, uyển chuyển, nhịp nhàng, cân đối trong lời nói. Xét ở đặc điểm này, phương thức hiệp gần gần giống với thành ngữ tiếng Việt. Ví dụ:

(28) Đề khó. Chán như con gián.

(29) - Bài khó mà bồ giải xong rồi á?

- Chuyện. Cao thủ đầu có mủ.

- Phương thức cải biên là phương thức dựa trên các thành ngữ, tục ngữ để tạo ra các ngữ mới lại của $\mathrm{SV}$, chẳng hạn: anh hùng bàn phím = anh hùng rơm, hồng nhan bạc phận = hồng nhan bạc tỉ, chân dài lưng ngắn = chân dài não ngắn, xấu nhu $m a=x a ̂ ́ u$ $n h u$ gấu,... Đây là phương thức thể hiện sự tiếp thu tinh hoa văn hoá của $S V$ bởi thành ngữ, tục ngữ là những tài sản mang đậm văn hoá của người Việt.

(30) Thôi. Học đi các ông mai thi rồi. Anh hùng bàn phím cho lắm. Mai thi rớt thì hay.

(31) Xấu nhur gấu lại còn điệu chảy mỡ.

- Phương thức sáng tạo mới.

Đây là phương thức mang đậm dấu ấn của nhóm SV trên các diễn đàn. Phương thức này sử dụng sự 
tương đồng về nghĩa để tạo ra các kết cấu mới sinh động, chẳng hạn: đắng lòng thanh niên, dậy thì thành công, năm trăm anh em, bó tay chấm com, giờ cao su, thông tấn xã con vịt, a cay con chim cú, anh hùng núp, fan cứng điểm danh, đau để trưởng thành, thân ái và quyết thắng,... Ví dụ:

(32) Mấy thánh nổ to giờ anh hùng núp hết à?

(33) Sau bốn năm, ẻm nó đã dậy thì thành công.

b) Về ngữ nghĩa

- Các kết cấu mới lạ không chỉ độc đáo về mặt ngữ âm mà con phong phú, đa dạng về ngữ nghĩa. Các kết cấu này về mặt thể hiện nghĩa được chia thành hai dạng:

+ Các kết cấu có đặc tính giống như các thành ngữ, tục ngữ thường mang nhiều nét nghĩa khác nhau và có tính biểu trưng cao. Chẳng hạn: anh hùng bàn phím, hồng nhan bạc tỉ,... các yếu tố trong kết cấu khá ổn định và mang tính biểu trưng cao.

+ Các kết cấu là các tổ hợp từ tự do như: đắng lòng thanh niên, dậy thì thành công, năm trăm anh em, anh hùng núp, fan cứng điểm danh, đau để truởng thành, thân ái và quyết thắng,... thường kết cấu lỏng lẻo và tính biểu trưng thấp hơn.

- Các kết cấu mới phản ánh tính dân tộc và tinh thần thời đại. SV Việt Nam trong thời hội nhập không chỉ thay đổi về chất mà còn có sự thay đổi về lượng. Thông qua các kết cấu này thể hiện nhịp sống xã hội, tư duy và phản ứng của nhóm sinh viên trước thay đổi của xã hội, chẳng hạn: dưới cách mạng công nghệ 4.0, cuộc sống và học tập của $\mathrm{SV}$ gắn chặt với các phương tiện công nghệ thông minh, tương tác rộng không chỉ nằm trong phạm vi nhóm nhỏ mà mở rộng ra trở thành các diễn đàn có số lượng vài nghìn người đến hàng chục nghìn người. Các kết cấu như: ăn online, ngủ online, sống online, mắt dán facebook, tay luoót bàn phím, đăng facebook, nghin like, bó tay chấm com,... trở thành những ngôn từ cửa miệng của SV. Ví dụ:

(34) Với trường hợp này, bó tay chấm com luôn.

(35) Nghe tin từ thông tấn xã con vịt thì có ngày ngỏm đó.

\section{Kết luận}

Tiếp cận ngôn ngữ mạng của sinh viên từ bình diện cấu trúc, chúng tôi dựa vào các lí thuyết về phương ngữ xã hội và ngôn ngữ mạng; sinh viên và bối cảnh văn hoá để tìm hiểu các đặc điểm ngôn ngữ mạng của sinh viên. Triển khai tìm hiểu đối tượng ở ba nội dung: tiếng lóng, chêm xen ngôn ngữ thứ hai trong tương tác và kết cấu mới lạ theo trật tự: về mặt ngữ âm (cấu tạo); về mặt từ vựng và về mặt ngữ nghĩa, chúng tôi đã lần lượt phân tích miêu tả và làm rõ từng đặc điểm. Từ kết quả nghiên cứu và phân tích khẳng định: tiếng lóng là hình thức phổ biến được sử dụng của SV trên mạng xã hội facebook (97 từ ngữ); chêm xen ngôn ngữ thứ hai trong tương tác (72 từ ngữ) và kết cấu mới lạ ít sử dụng (31 từ ngữ). Từ phân tích, khẳng định: Ngôn ngữ mạng của SV trên bình diện cấu trúc không chỉ phản ánh đặc trưng ngôn ngữ của nhóm đối tượng năng động, sáng tạo, dám nghĩ, dám làm, mang trên mình sứ mạng học tập để phụng sự tương lai dân tộc mà còn thể hiện sự vận động trong nhóm trước sự biến động của thời đại. Ngôn ngữ là một bộ phận của văn hoá xã hội, cũng là một trong những lĩnh vực thể hiện sự lệch chuẩn, sáng tạo, khẳng định mình.

\section{REFERENCES}

[1] Fasold Ralph, The Sociolinguistics of Society, Oxford - Brasil, Blackwell Ltd, 1990.

[2] Nguyen Van Khang, Social Linguistics, Education Publishing House, Hanoi, 2012.

[3] Nguyen Van Khang, Language in social networks , Hong Duc Publishing House, 2019.

[4] National Assembly of the Socialist Republic of Vietnam, Law on Higher Education, National Assembly of the 13th Socialist Republic of Vietnam, Term XIII, in the 3rd session, 2012

[5] Nguyễn Sanh Phúc, English Vietnamese dictionary, Dong Nai Publishing House, 1995.

[6] Do Huu Chau, General Linguistics Pragmatics (Volume 2), Education Publishing House, Hanoi, 2001.

[7] Huu Dat, Culture and language of communication of the Vietnamese, Culture Information Publishing House, Hanoi, 2000.

[8] Bui Minh Toan, Word in communication, Education Publishing House, Hanoi, 1999.

[9] Gumperz, J. J., Language in Social Groups, Standford: Standford University Press, 1971.

[10] Labov, W., The Study of Language in its Social Context, Studium Generale, 23:30- 87, 1970.

[11] Language data: https://www.facebook.com/sinhvienfanpage;

https://www.facebook.com/KenhSV.vn; https://www.facebook.com/hoisinhvien.com.vn; https://www.facebook.com/vnu.ulis; ;ttps://www.face book.com/Đoàn-thanh-niên-Hội-sinh-viên-trườngĐại-học-Tân-Trào; https://www.facebook.com/honghotsinhvien 


\section{SOCIAL NETWORKING LANGUAGE CHARACTERISTICS OF STUDENTS}

FROM THE STRUCTURE DIMENSION (THROUGH SOME FORUMS UNDER THE FACEBOOK SOCIAL NETWORK)

\begin{tabular}{l}
\hline Article info \\
\hline Recieved: \\
05/7/2020 \\
Accepted: \\
20/9/2020 \\
\hline
\end{tabular}

Keywords: social network language, students, structure dimension, forum.

\begin{abstract}
This article is based on theories related to social dialects and social network languages; student and cultural background to learn the characteristics of the social network language of students from the structure dimension in aspects such as slang, interleaving the second language in interaction, new textures. From the research results, we assert that: The social network language of students on the structure dimension shows the dynamism, creativity, dare to think and dare to do of youth and the movement of the era is expressed through students' language.
\end{abstract}

